\title{
НИЗКОТЕМПЕРАТУРНЫЕ СПЕКТРЫ КОМБИНАЦИОННОГО РАССЕЯНИЯ ПРУСТИТА
}

K. HALLER, LJUBOV REBANE, J. VOSSOTSANSKI, V. SLIVKA. PRUSTIIDI KOMBINATSIOONHAJUMISSPEKTRID MADALATE TEMPERATUURIDE KORRAL

K. HALLER, LYUBOV REBANE, J. VYSSOTCHANSKI, V. SLIVKA. LOW TEMPERATURE RAMAN SPECTRA OF A PROUSTITE CRYSTAL

Разнообразные физические свойства кристалла прустита $\left(\mathrm{Ag}_{3} \mathrm{AsS}_{3}\right)$ делают его ценным материалом для квантовой электроники. По данным $\left.{ }^{1}\right]$, в области температур от 4,2 до $300 \mathrm{~K}$ прустит имеет два фазовых перехода (ФП): I рода при $24 \mathrm{~K}$ и II рода при $56 \mathrm{~K}$. Высокотемпературная фаза $(T>56 \mathrm{~K})$ имеет пространственную группу $C_{3 v}^{6} \equiv R 3 c \quad$ с двумя структурными единицами в элементарной ячейке $\left[{ }^{2}\right]$ (тригональная сингония). Эта фаза изучена разными физическими методами (см., напр., $\left.\left[{ }^{3-9}\right]\right)$, в том числе и методом комбинационного рассеяния (KР) света $\left[{ }^{10,11}\right]$. Теоретико-групповой анализ нормальных колебаний тригональной фазы показал 19 активных в КР мод $\left(6 A_{1}+13 E\right)$. Проведенное в $\left[{ }^{10}\right]$ рассмотрение симметричных координат единичной ячейки показало, что нормальные колебания $338 \mathrm{~cm}^{-1}$ $\left(A_{1}\right)$ и $366 \mathrm{~cm}^{-1}\left(A_{1}\right)$ включают в основном валентное колебание тетраэдра $\mathrm{AsS}_{3}^{3-}$, а нормальное колебание $143 \mathrm{~cm}^{-1}\left(A_{1}\right)$ включает в основном деформационное колебание тетраэдра.

В настоящей работе приведены первые данные о спектрах КР в двух низкотемпературных фазах.

Спектры КР измерены с помощью двойного монохроматора Spex 1402 и детектированием в режиме счета фотонов с последующим накоплением сигнала в многоканальный анализатор. Описание экспериментальной установки приведено в $\left[{ }^{12}\right]$. Кристаллы выращивали методом Бриджмена $\left[{ }^{13}\right]$. Образец вырезался из кристалла, ориентированного с помощью рентгеноструктурного анализа при комнатной температуре (фаза $C_{3 v}^{6}$ ) таким образом, чтобы полярная ось $C$ была перпендикулярна к одной из граней прямоугольного параллелепипеда и лежала в плоскости другой грани.

Возбуждение осуществлялось лазерными линиями $6328 \AA(\mathrm{He}-\mathrm{Ne})$, 6471 и $6764 \AA(\mathrm{Kr})$ с мощностью до 50 мвт в линии. Ввиду сильного температурного сдвига края собственного поглощения прустита $\left[{ }^{4}\right]$ линия $6764 \AA$ использовалась при комнатной температуре, а коротковолновые линии - при низких температурах. Если не считать слабого эффекта реабсорбции, спектр КР не зависел от частоты возбуждения. Измере- 


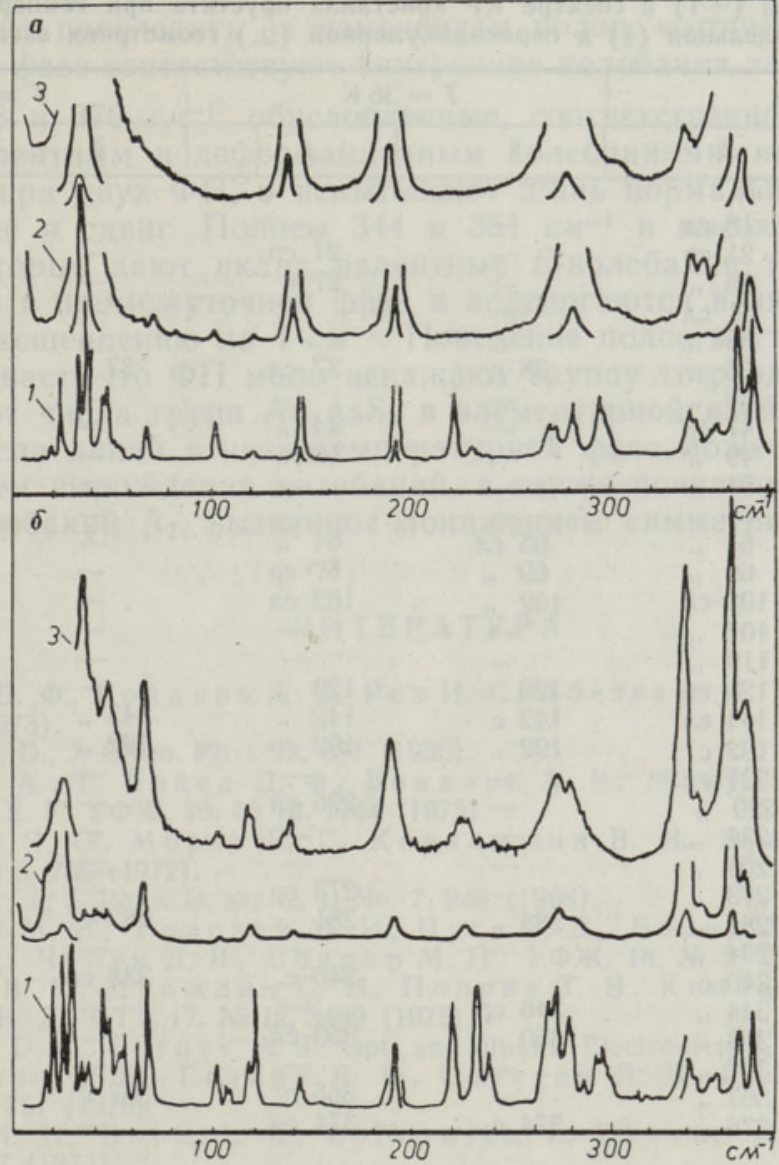

Спектры КР кристалла прустита (при параллельной (a) и перпендикулярной (б) поляризациях) в трех фазах при температурах 5 (1), 36 (2) и $100 \mathrm{~K}$ (3) (ширина щели 0,$65 ; 1,2$ и $1,2 \mathrm{~cm}^{-1}$ соответственно). Интенсивности всех спектров приведены в сравнимом масштабе (для наиболее сильных линий масштаб уменьшен в 10 раз, а масштаб спектра 1,6 увеличен в 10 раз).

ния проводились в области температур от 4,2 до $300 \mathrm{~K}$ в криостате типа УТРЕКС, где температура поддерживалась с точностью не хуже 0,05 град.

На рисунке показаны спектры КР при температурах 100 , 36 и 5 K, что соответствует трем разным фазам. Две геометрии эксперимента $\|[x(z z) y]$ и $\perp[x(z x) y]$ обозначены исходя из тригональной фазы (проявляются $A_{1}(\mathrm{TO})$ и $E(\mathrm{LO}+\mathrm{TO})$ моды соответственно). В таблице собраны положения всех зарегистрированных максимумов.

Измеренный при $100 \mathrm{~K}$ спектр КР тригональной фазы (спектр 3 на рисунке) в основном совпадает с приведенным в $\left[{ }^{10}\right]$. Дополнительно наблюдаются полосы при $21(E)$ и $35(E){C M^{-1}}^{-1}$ и на месте полосы $275(E) \quad c M^{-1}$ размещается дублет. В результате число наблюдаемых полос $\left(6 A_{1}+16 E\right)$ превышает предсказанное на основе теоретикогруппового анализа.

Переход к низкотемпературным фазам сопровождается увеличением 
Положения линий $( \pm 1)$ в спектре КР кристалла прустита при температурах 5,36 и $100 \mathrm{~K}$ при параллельной $(\|)$ и перпендикулярной $(\perp)$ геометриях эксперимента, $c M^{-1}$

\begin{tabular}{|c|c|c|c|c|c|c|}
\hline \multicolumn{3}{|c|}{$T=5 \mathrm{~K}$} & \multicolumn{2}{|c|}{$T=36 \mathrm{~K}$} & \multicolumn{2}{|c|}{$T=100 \mathrm{~K}$} \\
\hline & $\|$ & $\perp$ & $\|$ & $\perp$ & II & $\perp$ \\
\hline & сл" & $18 \mathrm{cл}$ & - & - & - & - \\
\hline & $\mathrm{cp}^{* *}$ & $21 \mathrm{cp}$ & - & $21 \overline{\mathrm{cp}}$ & - & $21 \bar{c}$ \\
\hline 26 & , & $26 \ldots$ & - & $27 \mathrm{c}$ & - & 26, \\
\hline $3 \overline{5}$ & , & $\begin{array}{l}31 \text { сл } \\
35 \%\end{array}$ & $\overline{-}$ & I. & I & 二 \\
\hline 38 & , & $38 "$ & $38 \mathrm{c}$ & 37 сл & $37 \mathrm{c}$ & $37 \mathrm{cp}$ \\
\hline & , & $39 \%$ & - & - & - & - \\
\hline $\begin{array}{l}47 \\
49\end{array}$ & $"$ & $\begin{array}{l}47 \mathrm{cp} \\
49\end{array}$ & - & $\begin{array}{l}44 ", \\
50\end{array}$ & . $=$ & $50^{-}$ \\
\hline $\begin{array}{l}49 \\
54\end{array}$ & сл & $54 ”$ & - & 00. & $\overline{-}$ & 50 _" \\
\hline 56 & $"$ & $56 "$ & - & - & - & - \\
\hline $6 \overline{9}$ & $\mathrm{cp}$ & $\begin{array}{l}65, ", \\
69,\end{array}$ & $65 \mathrm{cлl}$ & $64 \quad$ & $\overline{-}$ & $68 \overline{-}$ \\
\hline 102 & $"$ & 102 сл & $\begin{array}{r}69 " \\
102 ”\end{array}$ & $\begin{array}{r}67 \text { ср } \\
102 \text { сл }\end{array}$ & $I$ & 00 \\
\hline $11 \overline{9}$ & c7 & 107 ", & - & - & \pm & $107 \mathrm{cr}$ \\
\hline $\begin{array}{l}119 \\
121\end{array}$ & 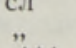 & 121 cp & 120 & 120 & $1-$ & $123 \overline{ }$ \\
\hline 144 & $c^{* * *}$ & $144 \mathrm{cл}$ & $142 \mathrm{c}$ & $142 "$ & 143 , & $143 \mathrm{cp}$ \\
\hline $\begin{array}{l}192, \\
221,\end{array}$ & ср & $\begin{array}{l}192 \mathrm{c} \\
221 \mathrm{cp}\end{array}$ & 192 „” & $192 \mathrm{cp}$ & $192 \ldots$ & $196 \ldots$ \\
\hline 229 & $c \pi$ & 229 , & E & 226 сл & - & $232 "$ \\
\hline $\begin{array}{l}238 \\
269\end{array}$ & $\ddot{c p}$ & $\begin{array}{l}238, " \\
269, "\end{array}$ & E & - & - & E \\
\hline 273 & $"$ & $273 "$ & - & $273 \mathrm{cp}$ & - & - \\
\hline 281 , & $"$ & $281 "$ & 283, & 281 & 282 c & $280 "$ \\
\hline $\begin{array}{l}295 \\
340\end{array}$ & , & $\begin{array}{l}294 \text { ” } \\
340 \text { c }\end{array}$ & E & $340 \bar{c}$ & $338 \mathrm{cp}$ & $286 \ldots$ \\
\hline 344 , & $"$ & 344 , & 346 ср & - & - & $344 \bar{c}$ \\
\hline 353 , & $"$ & $353 "$ & 350, & 350 сл & - & $351 \mathrm{cp}$ \\
\hline $\begin{array}{l}35 \%, \\
367\end{array}$ & $"$ & $\begin{array}{l}357 " \\
367 ”\end{array}$ & - & $365 \bar{c}$ & $367 \mathrm{c}$ & $367 \overline{\mathrm{c}}$ \\
\hline & c & $376 \%$ & $374 \mathrm{c}$ & $374 \mathrm{cp}$ & - & 374, \\
\hline
\end{tabular}

*сл - слабая линия, ${ }^{* *} \mathrm{cp}-$ средняя линия, ***c - сильная линия.

числа линий и уменьшением интенсивности линий перпендикулярной поляризации. При переходе в самую низкотемпературную фазу интенсивность спектра перпендикулярной поляризации, за исключением области $330-380$ cм $^{-1}$ валентных колебаний $\mathrm{AsS}_{3}^{3-}$, падает на порядок (ср. спектры $a$ и б) без заметного перераспределения интенсивности. ФП II рода сопровождается изменениями в спектре KР (ср. спектры 2 и $3, a$ и б). В спектре параллельной поляризации вместо полосы 338 $c \mu^{-1}$ появился дублет 340 и $350 \mathrm{~cm}^{-1}$. В результате сужения интенсивной полосы $38 \mathrm{~cm}^{-1}$ стали видны две слабые полосы 64 и $67 \mathrm{~cm}^{-1}$. Интенсивная полоса $282 \mathrm{~cm}^{-1}$ не испытала сужения и приняла сложный неэлементарный контур. Полосы 102 и 120 см $^{-1}$ приобрели слабую параллельную составляющую, которая в низкотемпературной фазе еще усиливается. В перпендикулярной поляризации помимо общего ослабления интенсивности сильно ослабляется полоса $50 \mathrm{~cm}^{-1}$.

При ФП I рода в низкотемпературной фазе (спектры $1, a$ и б) появляются 8 новых полос и 7 полос испытывают дублетное расщепление (см. таблицу). Следует отметить, что в низкотемпературной фазе большинство колебаний проявляются как в параллельной, так и в перпендикулярной составляющих. «Чисто» перпендикулярную поляризацию имеют только три полосы 31,65 и $107 \mathrm{~cm}^{-1}$. 
Интересно проследить за изменением полос, которым в высокотемпературной фазе соответствуют внутренние колебания тетраэдра $\mathrm{AsS}_{3}^{3-}$. Полосы 143 и 376 см $^{-1}$, обусловленные, соответственно, полносимметричным валентным и деформационным колебаниями, не претерпевают изменений при двух ФП, а испытывают лишь нормальное температурное сужение и сдвиг. Полосы 344 и $351 \mathrm{~cm}^{-1}$ в высокотемпературной фазе, в которые дают вклад валентные $E$-колебания тетраэдра, мало изменяются в промежуточной фазе и подвергаются в низкотемпературной фазе расщеплению на $4 \mathrm{~cm}^{-1}$. Поведение полос внутренних колебаний показывает, что ФП мало искажают группу тетраэдра, а также не увеличивают числа групп $\mathrm{Ag}_{3} \mathrm{AsS}_{3}$ в элементарной ячейке. Резкое увеличение числа линий в низкотемпературной фазе может быть обусловлено снятием вырождения колебаний, а также появлением ранее неактивных колебаний $\mathrm{A}_{2}$, вызванное понижением симметрии решетки.

\section{ЛИ Т Е Р А Т У Р А}

1. Байса Д. Ф., Бонд арь А. В., Рез И. С., Абезгауз А. И., УФЖ, 18, № 9, 1550 (1973).

2. Harker, D., J. Chem. Phys., 9, 381 (1936).

3. Беляев А. Д., Байса Д. Ф., Бондарь А. В., Мачулин В. Ф., Мисе люк Е. Г., УФЖ, 20, № 10, 1744 (1975).

4. Довгий Я. О., Мороз Е. Г., Коралышин В. Н., Буцко Н. Н., УФЖ, 17, № 5, 766 (1972).

5. W a rker, J., J. Phys. D, ser. 2, 1, No. 7, 949 (1968).

6. Гурзан М. И., Головей М. И., Пуга Г. Д., Борец А. Н., Туряница И. Д., Че пур Д. В., Бонд а М. П., УФЖ, 18, № 2, 274 (1973).

7. Новик В. К., Дрождин С. Н., Попова Т. В., Копцик В. А., Гаври л о в а Н. Д., ФТТ, 17, № 12, 3499 (1975).

8. Hanna, D. C., T urner, A. J., Opt. and Quant. Electronics, 8, 213 (1976).

9. Бредихин В. И., Генкин В. Н., Соустов Л. В., Квант. электроника, 3, № 4, 751 (1976).

10. B yer, H. H., B obb, L. C., Lefkowitz, I., Deaver, B. S., Ferroelectrics, $5,207(1973)$.

11. Riccius, H. D., Carey, P. R., Siimann, O., phys. stat. sol. (b) 72 , K99 (1975).

12. Ребане Л. А., Хальдре Т. Ю., Новик А. Е., Гороховский А. А., ФТТ, 15, № 11, 3188 (1973).

13. Holovey, M. I., Ole xe y k, I. D., Curzan, M. I., Rez, I. S., P a n y ko, V. V., Vorochilov, Yu. V., Rigan, M. Yu., Canegev, I. G., Bogdano" va, A. V., Kristall und Technik, 6, N 5, 631 (1971). 